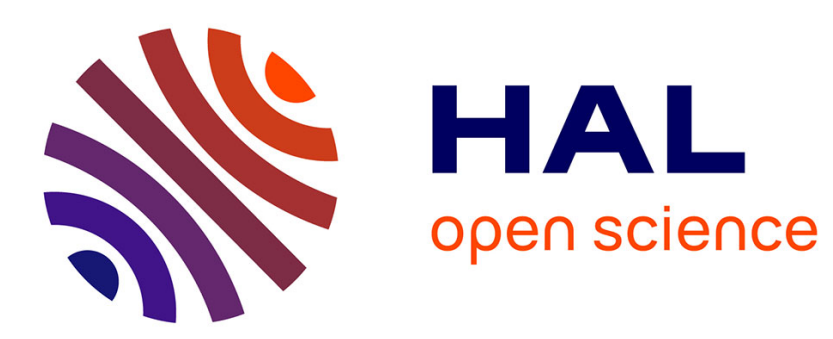

\title{
Prescribed-time predictor control of LTI systems with input delay
}

\author{
Nicolás Espitia, Wilfrid Perruquetti
}

\section{To cite this version:}

Nicolás Espitia, Wilfrid Perruquetti. Prescribed-time predictor control of LTI systems with input delay. 59th IEEE Conference on Decision and Control (CDC)., Dec 2020, Jeju Island (virtual), South Korea. hal-03021401

\section{HAL Id: hal-03021401 https://hal.science/hal-03021401}

Submitted on 24 Nov 2020

HAL is a multi-disciplinary open access archive for the deposit and dissemination of scientific research documents, whether they are published or not. The documents may come from teaching and research institutions in France or abroad, or from public or private research centers.
L'archive ouverte pluridisciplinaire HAL, est destinée au dépôt et à la diffusion de documents scientifiques de niveau recherche, publiés ou non, émanant des établissements d'enseignement et de recherche français ou étrangers, des laboratoires publics ou privés. 


\section{Prescribed-time predictor control of LTI systems with input delay}

\author{
Nicolás Espitia
}

\author{
Wilfrid Perruquetti
}

\begin{abstract}
This paper deals with the problem of prescribedtime stabilization of controllable linear systems with input delay. The problem is reformulated under a cascade PDE-ODE setting from which a prescribed-time predictor feedback is designed based on the backstepping approach, and whose transformation makes use of time-varying kernels. The bounded invertibility of the transformation is guaranteed. It is proved that the solution converges to the equilibrium in a prescribed-time. A simulation example is presented to illustrate the results.
\end{abstract}

\section{INTRODUCTION}

Finite, fixed-time and prescribed-time stabilization and estimation have been extensively considered in the framework of linear and nonlinear ordinary differential equations (ODEs) (see e.g. [9], [4], [20], [16], [11], [21], [10], [13]). For infinite dimensional systems, namely partial differential equations (PDEs), these non-asymptotic concepts have been of great interest and some contributions can be highlighted for 1D hyperbolic and parabolic PDEs: see e.g. [19], [5], [2], [5], [7], [6], [8], [22], [20]. For systems in both finite and infinite dimension, the need to meet some performance, time constraints and precision has highly motivated the stabilization and estimation in finite, fixed and prescribedtime.

In the framework of time-delay systems, however, finite, fixed and prescribed-time concepts have not achieved a sufficient level of maturity and are still challenging topics. Most of the results on stabilization and estimation are based on asymptotic or exponential guarantees. In particular, the exponential stabilization of LTI systems with input delay and linear time-varying systems with delays can be performed based on the predictor feedback and model reduction-based techniques (see.e.g [15], [14], [3], [17]. One of the pioneering contributions for stabilization in finite-time for time-delay systems are e.g. [12] and [18], the latter having pointed out some key obstructions for the design of static finite-time controllers and having therefore come up with a controller which is based on the Artstein's transformation [1] to stabilize in finite-time (with a settling time depending on initial conditions of the system). Nevertheless, to the best of our knowledge, prescribed-time stabilization for LTI systems with input delay has not been studied yet in the literature. The main feature of this type of non-asymptotic concept is that time of convergence can be prescribed in the design independently of initial conditions. Therefore, in this paper, we provide new results for the the problem of prescribed-time stabilization

*This work has been partially supported by ANR Project Finite4SoS (ANR 15-CE23-0007). N. Espitia and W. Perruquetti are with CRIStAL UMR 9189 CNRS - Centre de Recherche en Informatique Signal et Automatique de Lille - CNRS, Centrale Lille, Univ. Lille, F-59000 Lille, France. Corresponding author: nicolas.espitia-hoyoseuniv-lille.fr of controllable linear systems with input delay. We rely on a PDE-ODE cascade setting and on the use of an invertible Volterra backstepping transformation whose kernels are timevarying. We propose a target system which is prescribedtime stable. This stability property can be related back to the original system as long as the backstepping transformation is bounded invertible. The resulting controller (predictor-like) contains time-varying functions as the kernels turn out to be time varying. We call it then prescribed-time predictor control. In order to better communicate the key ideas of our approach, we focus mainly on a simple linear scalar equation with input delay, while being actually the main contribution of this paper. An overview on the extension to more general LTI systems with input delay is briefly presented.

This paper is organized as follows. In Section II, we introduce the LTI system with input delay and some preliminaries on prescribed-time stability. In Section III, we use a PDE-ODE setting under a backstepping approach to come up with a prescribed-time predictor controller. We focus on the scalar case to better communicate the main ideas of our approach. Then, an overview of the generalization to a LTI systems is provided in Section IV. In Section $\mathrm{V}$ we consider a numerical example to illustrate the results. Finally, conclusions and perspectives are given in Section VI.

The proofs of some results are omitted (some others are sketched) due to space limitation.

Notations: $\mathbb{R}_{+}$will denote the set of nonnegative real numbers. For non zero integers $m$ and $n$, let $0_{m \times n}$ be the $(m, n)$-matrix with zero entries, $I_{m}$ be the identity matrix of dimension $m, J_{n}=\left(\left(0_{(n-1) \times 1}, I_{n-1}\right)^{\top}, 0_{n \times 1}\right)^{\top}$ (Jordan matrix $)$ and $\mathcal{L}_{n}(p)=\left(0_{(n) \times(n-1)}, p\right)^{\top}$, where $p \in \mathbb{R}^{n}$. $L_{m}^{(\alpha)}(\cdot)$ denotes the generalized Laguerre polynomials and $\sigma_{n}(\cdot)$ denotes the elementary symmetric polynomials. The set of all functions $g:[0, h] \rightarrow \mathbb{R}^{n}$ such that $\int_{0}^{h} g(x)^{2} d x<$ $\infty$ is denoted by $L^{2}\left((0, h), \mathbb{R}^{n}\right)$. A continuous function $\alpha:[0, a) \subset \mathbb{R}_{+} \rightarrow \mathbb{R}_{+}, r \mapsto \alpha(r)$, is said to be a class- $\mathcal{K}$ function if it is strictly increasing with $\alpha(0)=0$. $\alpha$ is a class- $\mathcal{K}_{\infty}$ function if it is a class- $\mathcal{K}$ function with $a=\infty$ and $\alpha(r) \rightarrow \infty$ as $r \rightarrow \infty$. A continuous function $\beta:[0, a) \subset \mathbb{R}_{+} \times \mathbb{R} \rightarrow \mathbb{R}_{+},(r, t) \mapsto \beta(r, t)$, belongs to class$\mathcal{K} \mathcal{L}$ if for each fixed $t$, the mapping $r \mapsto \beta(r, t)$ belongs to class $\mathcal{K}_{\infty}$ with respect to $r$; and, for each fixed $r \in \mathbb{R}_{+}$, the mapping $t \mapsto \beta(r, t)$ is decreasing with respect to $t$ and $\lim _{t \rightarrow+\infty} \beta(t, r)=0$. 


\section{PROBLEM STATEMENT AND PRELIMINARIES ON PRESCRIBED-TIME STABILTY}

Let us consider the following LTI system with input delay:

$$
\dot{z}(t)=A z(t)+B u(t-h), z \in \mathbb{R}^{n}, u \in \mathbb{R},
$$

where $h>0$ is a known constant delay. $(A, B)$ are matrices of appropriate dimensions and satisfy the Kalman rank controllability condition for LTI systems. Thus $(A, B)$ can be assumed to be in the canonical controllability form without loss of generality: this is $A=J_{n}+\mathcal{L}_{n}(a), a=$ $\left(a_{0}, \ldots, a_{n-1}\right)^{\top}, B=(0, \ldots, 0,1)^{\top}$. We assume $u(t)=$ $0, \forall t \in[-h, 0)$. The goal is to design a control (predictortype) achieving "uniform fixed-time stability in a prescribed time" (in short $U \operatorname{PrTS}$ ). In order to better communicate the key ideas for the design of the prescribed-time predictor control for system (1), we are going to deal first with some preliminary results of prescribed time stability and the analysis and design for a simple linear scalar equation with input delay.

Let us first recall the following general definition of UPrTS (see e.g [21]).

Definition 1: The origin of the system $\dot{z}=f(t, z)$ is said to be UPrTS if there exist a class $\mathcal{K} \mathcal{L}$ function $\beta$ and a function $\mu:\left[t_{0}, t_{0}+T\right) \rightarrow \mathbb{R}_{+}$such that $\mu$ tends to infinity as $t$ goes to $t_{0}+T$ and, $\forall t \in\left[t_{0}, t_{0}+T\right)$

$$
\|z(t)\| \leq \beta\left(\left\|z\left(t_{0}\right)\right\|, \mu\left(t-t_{0}\right)\right) .
$$

We study the previous stability property on a scalar linear time-varying autonomous system without delay. The next lemma gives a sufficient condition for UPrTS of the following scalar equation:

$$
\dot{z}(t)=-c(t) z(t), \quad z\left(t_{0}\right)=z_{0} .
$$

Lemma 1: Let $c \in L^{1}(\mathbb{R})$ with $c(t)>0$ (a.e on $\mathbb{R}$ ), then (2) is UPrTS with a prescribed time $T>0$ if $\lim _{t \rightarrow t_{0}+T} \bar{c}(t)=+\infty$ where $\bar{c}(t)=\int_{t_{0}}^{t} c(\tau) d \tau$.

In what follows, we consider the following blow-up function defined on $\left[t_{0}, t_{0}+T\right)$ :

$$
c(t)=\frac{\left(c_{0} T\right)^{2}}{\left(T+t_{0}-t\right)^{2}}, \quad c\left(t_{0}\right)=c_{0}^{2},
$$

Notice that (2) is UPrTS (in light of Lemma 1).

\section{PRESCRIBED TIME PREDICTOR CONTROL: PDE BACKSTEEPING APPROACH}

We follow a cascade PDE-ODE setting (i.e. cascade linear hyperbolic PDE with an LTI system) introduced in [15], [14] under the backstepping approach which makes uses of an invertible Volterra transformation. As we will see, the key idea is to transform the original system into a target system that is $\operatorname{UPrTS}$ and that meets the requirements for a convergence in a time $T+h+t_{0}$ where $T$ is fixed a priori, $h$ is the known input delay and $t_{0}$ is the initialization time (for simplicity, it will be selected as $t_{0}=0$ ).

As aforementioned, in order to better communicate the main ideas of our approach, we first study a scalar linear system with input delay. The extension to the $n$-dimensional case (i.e. LTI systems of the form (1)) follows as similar reasoning related to the backstepping-based method studied for the scalar case (which constitutes main keystone of this paper contribution) as well as some transformations in the framework of on liner time-varying systems. An overview of such an extension is given, in a very informative way, in Section IV. Indeed, the details of the proofs of the formulas as well as the analysis are not provided due to space limitation and require a complete and rigorous exposure which is beyond the scope of this paper.

\section{A. Scalar case}

Let us consider the following control system:

$$
\dot{z}(t)=a z(t)+b u(t-h),
$$

where $z \in \mathbb{R}, h \geq 0$ and $u(t)$ is the control input which is delayed $h>0$ units of time $(u(t)=0, \forall t \in[-h, 0))$. For our analysis and design, we assume that $T \geq h$. Following [15], [14], the system (4) can be rewritten as cascade PDE-ODE system:

$$
\begin{aligned}
\dot{z}(t) & =a z(t)+b \omega(t, 0) \\
\omega_{t}(t, x) & =\omega_{x}(t, x) \\
\omega(t, h) & =u(t),
\end{aligned}
$$

$t \geq 0, x \in[0, h]$ and $\omega(t, \cdot)$ is the transport PDE state whose solution is given by $\omega(t, x)=u(t+x-h)$. We aim at stabilizing (5) (in turn (4)) in a prescribed time.

1) Backstepping control design and time-varying kernels: The invertible Volterra integral transformation is chosen to depend on time. It is given as follows:

$$
\begin{gathered}
\zeta(t, x)=\omega(t, x)-\int_{0}^{x} q(t-h, x, y) \omega(t, y) d y \\
-\gamma(t-h, x) z(t)
\end{gathered}
$$

whose kernel functions $q$ and $\gamma$ are time-varying. Under (6), we want to transform (5) into the following target system:

$$
\begin{aligned}
\dot{z}(t) & =-c(t-h) z(t)+b \zeta(t, 0) \\
\zeta_{t}(t, x) & =\zeta_{x}(t, x) \\
\zeta(t, h) & =0,
\end{aligned}
$$

where $\zeta:[0, \infty) \times[0, h] \rightarrow \mathbb{R}$ is the transport PDE state and $c$ is given by (3).

Following the standard methodology to find the kernel equations and taking into account their time-dependence, it can be shown that the kernels of transformation (6) satisfy the following PDE system:

$$
\begin{aligned}
q_{x}(t-h, x, y)+q_{y}(t-h, x, y) & =q_{t}(t-h, x, y) \\
q(t-h, x, 0) & =b \gamma(t-h, x) \\
\gamma_{x}(t-h, x)-\gamma_{t}(t-h, x) & =a \gamma(t-h, x)
\end{aligned}
$$

where $q$ and $\gamma$ are defined on the domains domains, respectively $\mathcal{T}_{q}:\{(t, x, y): 0 \leq y \leq x \leq h, \quad 0 \leq t<T+h-x\}$ and $\mathcal{T}_{\gamma}:\{(t, x): 0 \leq x \leq h, \quad 0 \leq t<T+h-x\}$. 
Proposition 1: The system (8) has well-posed $\mathcal{C}^{\infty}$ solutions on $\mathcal{T}_{q}$ and $\mathcal{T}_{\gamma}$, given by

$$
\begin{gathered}
q(t-h, x, y)=-(a+c(t-h+x)) \exp (a(x-y)), \\
\gamma(t-h, x)=-\frac{(a+c(t-h+x))}{b} \exp (a x),
\end{gathered}
$$

where $c$ is defined by (3).

Proof: By the method of characteristics, one can derive that the solution of the linear hyperbolic equation $\gamma$ in (8) is as follows:

$$
\gamma(t-h, x)=\exp (a x) \Gamma(t-h+x),
$$

for some $\Gamma$ to be characterized in the sequel. In (6) by letting $x=0$, we get $\zeta(t, 0)=\omega(t, 0)-\gamma(t, 0) z(t)$ in conjunction with the fact that $\dot{z}(t)=a z(t)+b \omega(t, 0)=-c(t-h) z(t)+$ $b \zeta(t, 0)$, we obtain

$a z(t)+b \omega(t, 0)=-c(t-h) z(t)+b(\omega(t, 0)-\gamma(t, 0) z(t))$ yielding

$$
\gamma(t-h, 0)=\frac{-(a+c(t-h))}{b}
$$

Moreover, it holds:

$$
\Gamma(t-h+x)=\gamma(t-h+x, 0)=-\frac{(a+c(t-h+x))}{b}
$$

This yields:

$$
q(t-h, x, 0)=b \exp (a x) \Gamma(t-h+x),
$$

Consequently,

$$
q(t-h, x, y)=b \exp (a(x-y)) \Gamma(t-h+x) .
$$

2) Inverse transformation and time-varying kernels: The inverse transformation is given by

$$
\begin{gathered}
\omega(t, x)=\zeta(t, x)+\int_{0}^{x} m(t-h, x, y) \zeta(t, y) d y \\
+\bar{\gamma}(t-h, x) z(t)
\end{gathered}
$$

whose kernels can be shown to satisfy the following PDE system:

$$
\begin{aligned}
m_{x}(t-h, x, y)+m_{y}(t-h, x, y) & =m_{t}(t-h, x, y) \\
m(t-h, x, 0) & =b \bar{\gamma}(t-h, x) \\
\bar{\gamma}_{x}(t-h, x)-\bar{\gamma}_{t}(t-h, x) & =-c(t-h) \bar{\gamma}(t-h, x)
\end{aligned}
$$

defined on the domains $\mathcal{T}_{m}:\{(t, x, y): 0 \leq y \leq x \leq$ $h, \quad 0 \leq t<T+h-x\}$ and $\mathcal{T}_{\bar{\gamma}}:\{(t, x): 0 \leq x \leq$ $h, \quad 0 \leq t<T+h-x\}$.

Proposition 2: The system (17) has well-posed solutions $\mathcal{C}^{\infty}$ on $\mathcal{T}_{m}$ and $\mathcal{T}_{\bar{\gamma}}$ given by

$$
\begin{aligned}
& m(t-h, x, y)=-(a+c(t-h+x)) \\
& \quad \times \exp \left(c_{0} T \sqrt{c(t-h+y)}\right) \exp \left(-c_{0} T \sqrt{c(t-h+x)}\right)
\end{aligned}
$$

$$
\begin{aligned}
& \bar{\gamma}(t-h, x)=\frac{-(a+c(t-h+x))}{b} \\
& \quad \times \exp \left(c_{0} T \sqrt{c(t-h)}\right) \exp \left(-c_{0} T \sqrt{c(t-h+x)}\right) .
\end{aligned}
$$

and $c$ defined by (3).

Proof: It follows the same reasoning as in the proof of Proposition 1 with a slight adaptation to deal with a linear hyperbolic equation with time-varying source term. Indeed, by the method of characteristics one can obtain that the solution to (17) is given as follows:

$$
\begin{aligned}
\bar{\gamma}(t-h, x)= & \frac{-(a+c(t-h+x))}{b} \\
& \times \exp \left(-\int_{0}^{x} c(t-h+x-s) d s\right)
\end{aligned}
$$

Then,

$$
\begin{aligned}
m(t-h, x, y) & =-(a+c(t-h+x)) \\
& \times \exp \left(-\int_{0}^{x-y} c(t-h+x-s) d s\right)
\end{aligned}
$$

Using the fact that under (3), it holds that

$$
\begin{aligned}
& -\int_{0}^{x} c(t-h+x-s) d s \\
& =c_{0} T \sqrt{c(t-h)}-c_{0} T \sqrt{c(t-h+x)}
\end{aligned}
$$

and

$$
\begin{aligned}
& -\int_{0}^{x-y} c(t-h+x-s) d s \\
& =c_{0} T \sqrt{c(t-h+y)}-c_{0} T \sqrt{c(t-h+x)}
\end{aligned}
$$

then, (18),(19) follow. This concludes the proof.

Prescribed-time predictor control: From (6), at $x=h$, and using (11), (13),(15), the boundary control is then:

$$
u(t)=-\frac{(a+c(t))}{b} e^{a h}\left(z(t)+\int_{0}^{h} e^{-a y} b u(t-h+y) d y\right),
$$

where $u(t-h+y)=\omega(t, y)$. Equivalently, we have:

$$
u(t)=-\frac{(a+c(t))}{b}\left(e^{a h} z(t)+\int_{t-h}^{t} e^{a(t-\theta)} b u(\theta) d \theta\right)
$$

Or, from (16) at $x=h$, along with (19)-(18), the boundary control can equivalently be written as follows:

$$
\begin{gathered}
u(t)=-\frac{(a+c(t))}{b}\left(e^{c_{0} T \sqrt{c(t-h)}} e^{-c_{0} T \sqrt{c(t)}}\right) z(t) \\
\left.+\int_{0}^{h} e^{c_{0} T \sqrt{c(t-h+y)}} e^{-c_{0} T \sqrt{c(t)}} b \zeta(t, y) d y\right) .
\end{gathered}
$$


3) Stability analysis: We perform the stability analysis on the target system and then we establish the bounded invertibility of the transformations by a suitable norm equivalence.

Lemma 2: Let $c$ be given by (3) with $c_{0}, T>0$ fixed. Let $h>0$ be a known delay. Then, the target system (7) is UPrTS stable in the following sense: for any $z_{0} \in \mathbb{R}$ it holds, for all $t \in[h, T+h)$ :

$$
|z(t)|^{2} \leq \eta_{z} \exp \left(-c_{0} T \sqrt{c(t-h)}\right)\left|z_{0}\right|^{2}
$$

where $\eta_{z}=\exp \left(2 c_{0} T \sqrt{c(-h)}\right)$. Moreover,

$$
|z(t)|^{2} \rightarrow 0, \quad \text { as } t \rightarrow T+h
$$

Proof: We exploit the cascade nature of the chosen target system (7) along with the fact that the transport PDE $\zeta$ is fixed-time stable. After $t=h$, one has that $\zeta \equiv 0$ and then compute explicitly the solution $z(t)$ starting from $z(h)$ to get (27).

Proposition 3: For the backstepping transformations (6) and (16), the following estimates hold:

$$
\begin{aligned}
& \|\zeta(t, \cdot)\|_{L^{2}}^{2} \leq M_{q}(t-h)\|\omega(t, \cdot)\|_{L^{2}}^{2}+M_{\gamma}(t-h)|z(t)|^{2} \\
& \|\omega(t, \cdot)\|_{L^{2}}^{2} \leq N_{m}(t-h)\|\zeta(t, \cdot)\|_{L^{2}}^{2}+N_{\bar{\gamma}}(t-h)|z(t)|^{2}
\end{aligned}
$$

where

$$
\begin{aligned}
M_{q}(t-h) & = & 4\left(1+h \int_{0}^{h}\left(\int_{0}^{x}|q(t-h, x, y)|^{2} d y\right) d x\right) \\
M_{\gamma}(t-h) & = & 2 \int_{0}^{h} \gamma^{2}(t-h, x) d x \\
N_{m}(t-h) & = & 4\left(1+h \int_{0}^{h}\left(\int_{0}^{x}|m(t-h, x, y)|^{2} d y\right) d x\right) \\
N_{\bar{\gamma}}(t-h) & = & 2 \int_{0}^{h} \bar{\gamma}^{2}(t-h, x) d x
\end{aligned}
$$

Lemma 3: Let $N_{\bar{\gamma}}(t-h)$ be given by (34). Then, the following holds true:

$$
\lim _{t \rightarrow T+h} \exp \left(-c_{0} T \sqrt{c(t-h)}\right) N_{\bar{\gamma}}(t-h)=0 .
$$

Proof: [Sketch] Using (19), we have $N_{\bar{\gamma}}(t-h)=$ $2 \exp \left(c_{0} T \sqrt{c(t-h)}\right) \times \mathcal{I}(t, h)$ (where $\mathcal{I}$ is some integral depending on time $t$ and $t-h$ ). Then using Young's inequality we split the integral into two terms each of them is again split into two terms to get:

$$
\begin{aligned}
& \exp \left(-c_{0} T \sqrt{c(t-h)}\right) N_{\bar{\gamma}}(t-h) \leq \\
& \exp \left(c_{0} T \sqrt{c(0)}\right)\left(\mathcal{F}_{11}(t)+\mathcal{F}_{21}(t)+\mathcal{F}_{12}(t-h)+\mathcal{F}_{22}(t-h)\right)
\end{aligned}
$$

It can be shown that each term $\mathcal{F}_{i j}(\cdot)$ goes to zero in time either $T$ or $T+h$ yielding (35).

Theorem 1: Let $c$ be given by (3). Let $h>0$ be a known delay and $c_{0}>0, T>0$ fixed and such that $T \geq h$. Let $N_{\bar{\gamma}}$ be given by (34) and $\eta_{z}>0$ as in Lemma 2. Then, the solution of the closed-loop system (5) with prescribed-time predictor control (24) (or (26)) satisfies for any $z_{0} \in \mathbb{R}$ and for all $t \in[h, T+h)$ :

$$
\begin{aligned}
|z(t)|^{2}+ & \|\omega(t, \cdot)\|_{L^{2}}^{2} \leq \eta_{z}\left(N_{\bar{\gamma}}(t-h)+1\right) \\
& \times \exp \left(-c_{0} T \sqrt{c(t-h)}\right)\left|z_{0}\right|^{2}
\end{aligned}
$$

Moreover,

$$
|z(t)|^{2}+\|\omega(t, \cdot)\|_{L^{2}}^{2} \rightarrow 0, \quad \text { as } \quad t \rightarrow T+h
$$

and $|u(t)| \rightarrow 0$ as $t \rightarrow T$.

Proof: [Sketch] Using (30) in Proposition 3, it holds, for $t \in[0, T+h)$ that

$$
\begin{aligned}
|z(t)|^{2}+\|\omega(t, \cdot)\|_{L^{2}}^{2} & \leq N_{m}(t-h)\|\zeta(t, \cdot)\|_{L^{2}}^{2} \\
& +\left(N_{\bar{\gamma}}(t-h)+1\right)|z(t)|^{2}
\end{aligned}
$$

By Lemma 2 and the fact that $\|\zeta(t, \cdot)\|_{L^{2}} \rightarrow 0$ as $t \rightarrow h$ (as well as the fact that $N_{m}(t-h)<\infty$, for all $\left.t \in[0, h]\right)$, then it holds for $t \in[h, T+h)$,

$$
\begin{aligned}
|z(t)|^{2}+ & \|\omega(t, \cdot)\|_{L^{2}}^{2} \leq \eta_{z}\left(N_{\bar{\gamma}}(t-h)+1\right) \\
& \times \exp \left(-c_{0} T \sqrt{c(t-h)}\right)\left|z_{0}\right|^{2}
\end{aligned}
$$

Then, by Lemma 3, we finally obtain that $|z(t)|^{2}+$ $\|\omega(t, \cdot)\|_{L^{2}}^{2} \rightarrow 0$, as $t \rightarrow T+h$. It remains to show that $|u(t)| \rightarrow 0$. Indeed, from (26) it holds

$$
\begin{aligned}
& |u(t)| \leq \frac{|a+c(t)|}{b} \exp \left(-c_{0} T \sqrt{c(t)}\right) \exp \left(c_{0} T \sqrt{c(t-h)}\right)|z(t)| \\
& \quad+\|m(t-h, h, \cdot)\|_{L^{2}}\|\zeta(t, \cdot)\|_{L^{2}} .
\end{aligned}
$$

The term $\|m(t-h, h, \cdot)\|_{L^{2}}<\infty$ for all $t \in[0, h]$. Moreover, we use the fact that $\|\zeta(t, \cdot)\|_{L^{2}} \equiv 0$ from $t \geq h$. Therefore, by Lemma 2 , it holds, for $t \geq h$ :

$$
|u(t)| \leq \frac{|a+c(t)|}{b} \eta_{z} \exp \left(-c_{0} T \sqrt{c(t)}\right)\left|z_{0}\right|
$$

from which we can conclude that $|u(t)| \rightarrow 0$ as $t \rightarrow T$.

\section{EXTENSION TO LTI SYSTEMS WITH SINGLE INPUT DELAY}

In this section, we present an overview of the results concerning the general case for the design of the predictorfeedback prescribe time stabilization of (1). Consider the cascade PDE-ODE formulation of (1), i.e.

$$
\begin{aligned}
\dot{z}(t) & =A z(t)+B \omega(t, 0) \\
\omega_{t}(t, x) & =\omega_{x}(t, x) \\
\omega(t, h) & =u(t),
\end{aligned}
$$

1) Backstepping control design and time-varying kernels: The backstepping transformation is as follows:

$$
\begin{gathered}
\zeta(t, x)=\omega(t, x)-\int_{0}^{x} q(t-h, x, y) \omega(t, y) d y \\
-\gamma^{\top}(t-h, x) z(t)
\end{gathered}
$$

such that $\gamma^{\top}(t-h, x) z(t)=\sum_{i=1}^{n} \gamma_{i}(t-h, x) z_{i}(t)$. Under (44), system (43) is mapped into the following target system:

$$
\begin{aligned}
\dot{z}(t) & =C(t-h) z(t)+B \zeta(t, 0) \\
\zeta_{t}(t, x) & =\zeta_{x}(t, x) \\
\zeta(t, h) & =0,
\end{aligned}
$$

with $C(t-h)=J_{n}+\mathcal{L}_{n}(-p(t-h))$ where $p(t-h)=$ $\left(p_{0}(t-h), \ldots, p_{n-1}(t-h)\right)$ with functions $p_{i-1}, i=1, \ldots, n$ being defined by

$$
p_{0}(t-h)=\sigma_{n}\left(r_{1}, . ., r_{n}\right) c^{n}(t-h),
$$


and for $j=1, \ldots, n-1$,

$$
\begin{aligned}
& p_{j}(t-h)=\frac{(\sqrt{c(t-h)})^{n-j}}{\left(c_{0} T\right)^{n-j}} \\
& \times \sum_{k=j}^{n}(-1)^{k-j} \sigma_{n-k}\left(r_{1}, \ldots, r_{n}\right)\left(\begin{array}{c}
k-1 \\
j-1
\end{array}\right) \frac{k !}{j !}\left(c_{0} T \sqrt{c(t-h)}\right)^{n-k}
\end{aligned}
$$

where $c(\cdot)$ is given by (3), $r_{i}>0, r_{i} \neq r_{j}$ for $i \neq j$ in the range of $n$ and $\sigma_{n-k}(\cdot)$ are the elementary symmetric polynomials defined by

$$
\begin{aligned}
\sigma_{0}\left(r_{1}, \ldots, r_{n}\right) & =1, \\
\sigma_{k}\left(r_{1}, \ldots, r_{n}\right) & =\sum_{1 \leq i_{1} \leq i_{2} \leq \ldots i_{k} \leq n} r_{i_{1}} r_{i_{2}} \ldots r_{i_{k}}, \\
\sigma_{n}\left(r_{1}, \ldots, r_{n}\right) & =\prod_{i=1}^{n} r_{i},
\end{aligned}
$$

and $\sigma_{k}\left(r_{1}, \ldots, r_{n}\right)=0$, for $k \geq n$.

The Kernel PDE equations of transformation (44) are:

$$
\begin{aligned}
q_{x}(t-h, x, y)+q_{y}(t-h, x, y) & =q_{t}(t-h, x, y) \\
q(t-h, x, 0) & =\gamma^{\top}(t-h, x) B \\
\gamma_{x}(t-h, x)-I_{n} \gamma_{t}(t-h, x) & =A^{\top} \gamma(t-h, x),
\end{aligned}
$$

where $q$ and $\gamma$ are defined on the $\mathcal{T}_{q}$ and $\mathcal{T}_{\gamma}$.

Proposition 4: The system (51) has well-posed $\mathcal{C}^{\infty}$ solutions on $\mathcal{T}_{q}$ and $\mathcal{T}_{\gamma}$, given by

$$
\begin{aligned}
q(t-h, x, y) & =\Gamma^{\top}(t-h+x) \exp (A(x-y)) B, \\
\gamma(t-h, x) & =\exp \left(A^{\top} x\right) \Gamma(t-h+x),
\end{aligned}
$$

where

$\Gamma_{i}(t-h+x)=-\left(a_{i-1}+p_{i-1}(t-h+x)\right), \quad i=1, \ldots, n$

and functions $p_{i-1}$ are defined by (46), (47).

2) Inverse transformation and time-varying kernels: The inverse transformation is given by

$$
\begin{gathered}
\omega(t, x)=\zeta(t, x)+\int_{0}^{x} m(t-h, x, y) \zeta(t, y) d y \\
+\bar{\gamma}^{\top}(t-h, x) z(t)
\end{gathered}
$$

whose kernels satisfy the following PDE system:

$$
\begin{aligned}
m_{x}(t-h, x, y)+m_{y}(t-h, x, y) & =m_{t}(t-h, x, y) \\
m(t-h, x, 0) & =\bar{\gamma}^{\top}(t-h, x) B \\
\bar{\gamma}_{x}(t-h, x)-I_{n} \bar{\gamma}_{t}(t-h, x) & =C^{\top}(t-h) \bar{\gamma}(t-h, x),
\end{aligned}
$$

defined on the domains $\mathcal{T}_{m}$ and $\mathcal{T}_{\bar{\gamma}}$.

Proposition 5: The system (56) has well-posed solutions $\mathcal{C}^{\infty}$ on $\mathcal{T}_{m}$ and $\mathcal{T}_{\bar{\gamma}}$ given by

$$
\begin{gathered}
m(t-h, x, y)=\Gamma^{\top}(t-h+x) V(t-h+x) \\
\times \mathcal{D}(t-h+x) \mathcal{D}^{-1}(t-h+y) V^{-1}(t-h+y) B \\
\bar{\gamma}(t-h, x)=V^{-\top}(t-h) \mathcal{D}^{-1}(t-h) \\
\mathcal{D}(t-h+x) V^{T}(t-h+x) \Gamma(t-h+x)
\end{gathered}
$$

where

$$
\begin{aligned}
& \mathcal{D}(s) \\
& =\operatorname{diag}\left(\exp \left(-r_{1} c_{0} T \sqrt{c(s)}\right), \ldots, \exp \left(-r_{n} c_{0} T \sqrt{c(s)}\right)\right), \\
& \Gamma_{i}(t-h+x)=-\left(a_{i-1}+p_{i-1}(t-h+x)\right)
\end{aligned}
$$

$p_{i-1}$ defined by (46), (47), and $V$ is polynomial-based Vandermonde matrix given as follows:

$$
V(t)=\left(\begin{array}{ccc}
1 & \cdots & 1 \\
\left(\delta^{0} \rho_{1}\right)(t-h) & \cdots & \left(\delta^{0} \rho_{n}\right)(t-h) \\
\vdots & \ddots & \vdots \\
\left(\delta^{n-2} \rho_{1}\right)(t-h) & \cdots & \left(\delta^{n-2} \rho_{n}\right)(t-h)
\end{array}\right)
$$

with

$$
\begin{aligned}
& \left(\delta^{k} \rho_{i}\right)(t-h)= \\
& \frac{-r_{i} c(t-h)(\sqrt{c(t-h)})^{k}}{\left(c_{0} T\right)^{k}} k ! L_{k}^{(1)}\left(r_{i} c_{0} T \sqrt{c(t-h)}\right),
\end{aligned}
$$

\section{Prescribed-time predictor control}

From 44, at $x=h$, and using (52)-(54), the boundary control is then:

$$
\begin{aligned}
u(t)= & \Gamma^{\top}(t) \exp (A h) z(t) \\
& +\int_{0}^{h} \Gamma^{\top}(t) \exp (A(h-y)) B u(t-h+y) d y,
\end{aligned}
$$

where $u(t-h+y)=w(t, y)$. Using the inverse transformation, from (55), at $x=h$, the boundary control can equivalently be written as follows:

$u(t)=\Gamma^{\top}(t)\left(V(t) \mathcal{D}(t) \mathcal{D}^{-1}(t-h) V^{-1}(t-h)\right) z(t)+$
$\int_{0}^{h} \Gamma^{\top}(t)\left(V(t) \mathcal{D}(t) \mathcal{D}^{-1}(t-h+y) V^{-1}(t-h+y)\right) B \zeta(t, y) d y$.

\section{3) Stability result:}

Theorem 2: Let $c$ be given by (3) and let $r_{\min }=$ $\min _{i=1, \ldots, n}\left\{r_{i}\right\}$ with $r_{i}>0$ involved in (46)-(47). Let $h>0$ be a known delay and $c_{0}>0, T>0$ fixed and such that $T \geq h$. Let $N_{\bar{\gamma}}=2 \int_{0}^{h} \bar{\gamma}^{\top}(t-h, x) \bar{\gamma}(t-h, x) d x$ where $\bar{\gamma}$ is given by (58). Then, the solution of the closed-loop system (43) with prescribed-time predictor control (61) (or (62)) satisfies, for any $z_{0} \in \mathbb{R}^{n}$ and for all $t \in[h, T+h)$ :

$$
\begin{aligned}
\|z(t)\|^{2}+ & \|\omega(t, \cdot)\|_{L^{2}}^{2} \leq \eta_{z}\left(N_{\bar{\gamma}}(t-h)+1\right) P(\sqrt{c(t-h)}) \\
& \times \exp \left(-r_{\min } c_{0} T \sqrt{c(t-h)}\right)\left\|z_{0}\right\|^{2}
\end{aligned}
$$

for some $\eta_{z}>0$ and $P$ a positive polynomial in $\sqrt{c(t-h)}$. Moreover,

$$
\|z(t)\|^{2}+\|\omega(t, \cdot)\|_{L^{2}}^{2} \rightarrow 0, \quad \text { as } \quad t \rightarrow T+h
$$

and $|u(t)| \rightarrow 0$ as $t \rightarrow T$. 


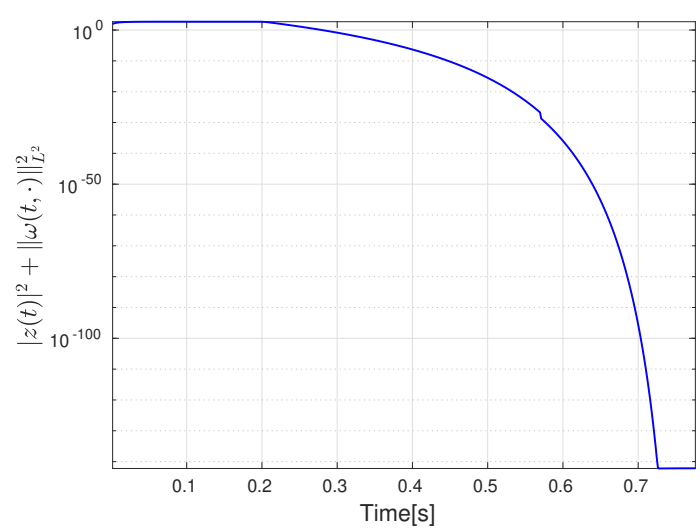

Fig. 1. Evolution of the $L^{2}$-norm of the closed-loop system (5) (logarithmic scale)

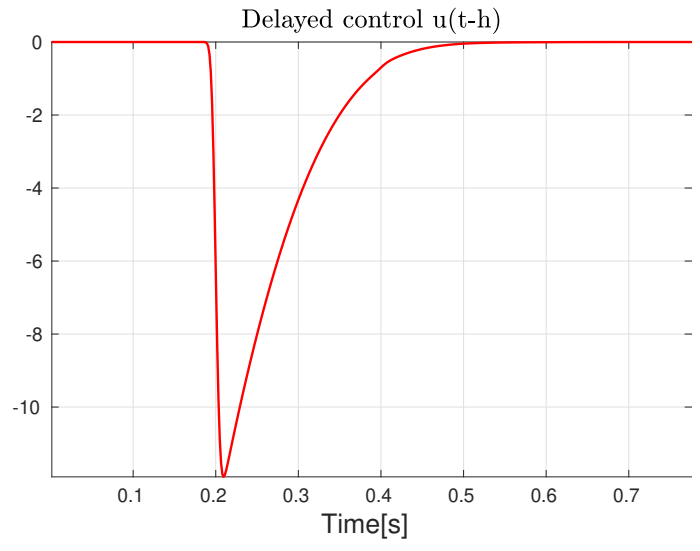

Fig. 2. Evolution of the delayed control (24).

\section{Simulations}

We consider a scalar linear equation with input delay (4). We borrowed the example from [18] with $a=1, b=\exp (h)$ and $h=0.2 \mathrm{~s}$. We fix $T=0.6 \mathrm{~s}$. Numerical simulations were done by discretizing the cascade PDE-ODE system (7) and making use of transformation (16). Figure 1 shows the evolution of the $L^{2}$-norm of the closed-loop system (plotted in logarithmic scale) with boundary control $u(t)$ (26). One can observe convergence to the origin as $t \rightarrow 0.8 \mathrm{~s}$. Figure 2 shows the time evolution of the delayed control.

\section{CONCLUSION}

We have addressed the problem of prescribed-time stability of linear systems with input delay. The key ideas are developed for delay compensation of a scalar linear equation with input delay. The prescribed-time predictor feedback design is carried out based on the backstepping approach which makes use of time-varying kernels, for which, the bounded invertibility of the backstepping transformation is guaranteed. An overview of the results for a more general case ( $n$-dimesnional LTI systems) is presented.

Future work includes prescribed-time stabilization of LTI systems with time-varying delay and observer-based predictor-feedback.

\section{REFERENCES}

[1] Z. Artstein. Linear systems with delayed controls: A reduction. IEEE Transactions on Automatic Control, 27:869-879, 1982.

[2] J. Auriol and F. Di Meglio. Minimum time control of heterodirectional linear coupled hyperbolic PDEs. Automatica, 71:300-307, 2016.

[3] N. Bekiaris-Liberis and M. Krstic. Compensation of time-varying input and state delays for nonlinear systems. Journal of Dynamic Systems, Measurement, and Control, 134(011009), 2012.

[4] S.P. Bhat and D.S. Bernstein. Finite time stability of continuous autonomous systems. SIAM J. Control Optim., 38(3):751-766, 2000.

[5] J.-M. Coron, L. Hu, and G. Olive. Finite-time boundary stabilization of general linear hyperbolic balance laws via fredholm backstepping transformation. Automatica, 84:95-100, 2017.

[6] J.-M. Coron and H.-M. Nguyen. Null controllability and finite time stabilization for the heat equations with variable coefficients in space in one dimension via backstepping approach. Archive for Rational Mechanics and Analysis, 225(3):993-1023, 2017.

[7] J. Deutscher. Finite-time output regulation for linear $2 \times 2$ hyperbolic systems using backstepping. Automatica, 75:54 - 62, 2017.

[8] N. Espitia, A. Polyakov, D. Efimov, and W. Perruquetti. Boundary time-varying feedbacks for fixed-time stabilization of constantparameter reaction-diffusion systems. Automatica, 103:398 - 407, 2019.

[9] V.T. Haimo. Finite time controllers. SIAM Journal of Control and Optimization, 24(4):760-770, 1986.

[10] J. Holloway and M. Krstic. Prescribed-time output feedback for linear systems in controllable canonical form. Automatica, 107:77-85, 2019.

[11] E. Jiménez-Rodríguez, AJ. Muñoz Vázquez, JD. Sánchez-Torres, M. Defoort, and AG. Loukianov. A Lyapunov-like characterization of predefined-time stability. IEEE Transactions on Automatic Control, pages $1-1,2020$.

[12] I. Karafyllis. Finite-time global stabilization by means of time-varying distributed delay feedback. SIAM J. Control Optim., 45:320-342, 2006.

[13] P. Krishnamurthy, F. Khorrami, and M. Krstic. A dynamic high-gain design for prescribed-time regulation of nonlinear systems. Automatica, 115:108860, 2020.

[14] M. Krstic. Delay Compensation for Nonlinear, Adaptive, and PDE Systems. Birkhäuser, 2009.

[15] M. Krstic and A. Smyshlyaev. Backstepping boundary control for first-order hyperbolic PDEs and application to systems with actuator and sensor delays. Systems \& Control Letters, 57(9):750-758, 2008.

[16] F. Lopez-Ramirez, A. Polyakov, D. Efimov, and W. Perruquetti. Finite-time and fixed-time observer design: Implicit lyapunov function approach. Automatica, 87:52 - 60, 2018.

[17] F. Mazenc, M. Malisoff, and S.-I. Niculescu. Reduction model approach for linear time-varying systems with delays. IEEE Transactions on Automatic Control, 59(2068-2081), 2014.

[18] E. Moulay, M. Dambrine, N. Yeganefar, and W. Perruquetti. Finitetime stability and stabilization of time-delay systems. Systems \& Control Letters, 57:561-566, 2008.

[19] V. Perrollaz and L. Rosier. Finite-time stabilization of $2 \times 2$ hyperbolic systems on tree-shaped networks. SIAM Journal on Control and Optimization, 52(1):143-163, 2014.

[20] A. Polyakov, D. Efimov, and W. Perruquetti. Finite-time and Fixedtime Stabilization: Implicit Lyapunov Function Approach. Automatica, 51(1):332-340, 2015.

[21] Y.-D. Song, Y.-J. Wang, J.-C. Holloway, and M. Krstic. Timevarying feedback for regulation of normal-form nonlinear systems in prescribed finite time. Automatica, 83:243 - 251, 2017.

[22] D. Steeves, M. Krstic, and R. Vazquez. Prescribed-time H1stabilization of reaction-diffusion equations by means of output feedback. In 2019 18th European Control Conference (ECC), pages 19321937, 2019 